\title{
Amelia of lower limb
}

INSERM

\section{Source}

INSERM. (1999). Orphanet: an online rare disease and orphan drug data base. Amelia of lower limb. ORPHA:294969

A rare, non-syndromic limb reduction defect characterized by complete or near-complete congenital absence of one (unilateral) or both (bilateral) of the lower extremities, occurring due to an intrauterine insult during the very early stages of embryonic development. It may be an isolated anomaly, but is more commonly observed in combination with multiple other congenital malformations. 